Doug Geisler, Eva K. Grebel, and Dante Minniti, eds.

\title{
Super Star Clusters in NGC 7673 and Mrk 8
}

\author{
N. Homeier \& J.S. Gallagher \\ University of Wisconsin-Madison, 475 N. Charter St., Sterling Hall, \\ Madison, WI 53706, USA
}

\begin{abstract}
.
We present an analysis of super star cluster populations using WFPC 2 on the Hubble Space Telescope for two starburst galaxies: NGC 7673 and Markarian 8. Both galaxies are luminious and have signatures of massive star formation, such as emission-line dominated optical spectra. Their optical appearances are highly disturbed, featuring prominent knots of intense star formation. Using HST F255W, F555W, and F814W images, we perform aperture photometry for a select sample of star clusters in different regions of each galaxy, and compare the results with evolutionary population synthesis models.
\end{abstract}

\section{Introduction}

Starburst galaxies are characterized by blue colors, strong nebular emission lines, and high surface brightnesses (Sargent 1970, Huchra 1977). In recent years interest in these objects has been motivated by their similarity to classes of galaxies found at moderate to high redshifts (Gallagher et al. 1989; Ellis 1997; Guzman et al. 1998). Starburst galaxies also have an important connection to more local phenomena, as it becomes increasingly clear that super star clusters (SSCs) are the predominant mode of star formation in these systems (Johnson et al. 1999; Hunter et al. 2000; Johnson \& Conti 2000).

Although the origin of globular clusters remains unclear, recent investigations (Ashman \& Zepf 1998) suggest that SSCs may be their progenitors. However, observations IMF truncation in some young star clusters (Brodie et al. 1998; Smith \& Gallagher 2001, this meeting) and theoretical support for such a truncation effect (Elmegreen 1997) may mean that SSCs do not have sufficient mass to remain bound for globular cluster lifetimes. Whatever their subsequent evolution, further investigation of the SSC mode of star formation is essential for our understanding of both field and cluster stellar populations.

\subsection{NGC 7673 \& Markarian 8}

The overall structure and kinematics of NGC 7673 were discussed by Homeier \& Gallagher (1999), who showed that, despite the disturbed optical appearance of this system, it is most likely a rotating disk galaxy seen nearly face-on. The starburst activity is within the inner half of the optical radius, concentrated in large complexes. The outer disk is relatively smooth and nearly circular, with ripples and wisps, and a shell feature at $21 \mathrm{kpc}$ from the optical center (Dettmar 


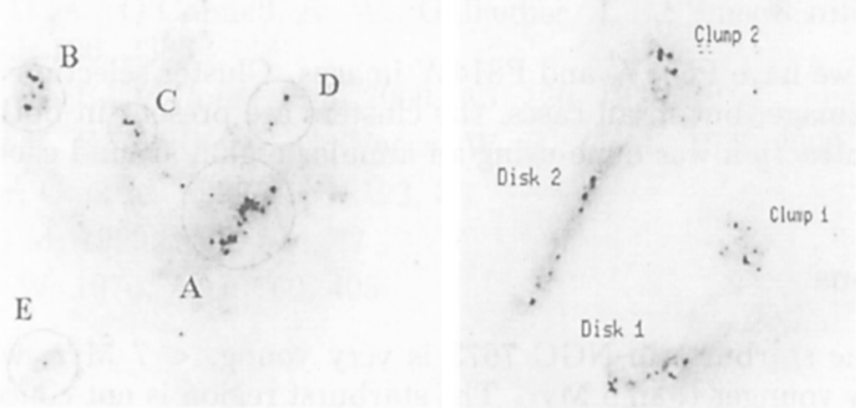

Figure 1. NGC 7673 on the left; Markarian 8 on the right. Both are HST WFPC2 images with the F555W filter.

etc. 1984 Homeier \& Gallagher 1999). Such features are likely remnants of a past interaction that triggered the starburst.

Markarian 8, along with NGC 7673, was assigned by Cassini \& Heidmann (1976) to a group of what they termed 'clumpy, irregular galaxies'. Further studies (refs) reveal Mrk 8 as a highly disturbed galaxy composed of (at least) 2 interacting disks with faint optical tidal tails (Esteban \& Mendez 1999). Integrated spectra of Mrk 8 shows broad stellar wind features, i.e. Mrk 8 is a Wolf-Rayet galaxy (Conti 1991, Esteban \& Mendez 1999).

\section{Method}

Regions and clusters in each galaxy were chosen with the intent of sampling areas in each galaxy with possibly different star formation histories, and not for a complete census of cluster formation. Further study of this nature is in progress for each of these galaxies.

Aperture photometry was performed with APPHOT. Aperture corrections and WF3 edge corrections were applied according to Holtzman et. al 1995. We compared cluster colors and magnitudes to evolutionary tracks from the Starburst99 models (Leitherer et al. 1999). The model track we present here is for a cluster mass of $10^{6} \mathrm{M} \odot$, an upper mass limit of $100 \mathrm{M} \odot$, and a metallicity of $\mathrm{z}=0.008$. Ages are marked in Myrs.

It should be noted that at the distances of these galaxies, $1 \mathrm{WF}$ pixel corresponds to $\sim 24 \mathrm{pc}$. Therefore, the objects we measure may be composites of smaller clusters, especially in crowded regions wuch as the center of NGC 7673.

\subsection{NGC 7673}

Cluster selection in each region was done first on the $\mathrm{F} 255 \mathrm{~W}$ image, as this has the lowest signal-to-noise. Background subtraction for the F555W and F814W images was problematic in some areas due to crowding. We experimented with various methods of background subtraction, and clusters marked with stars indicate a sensitivity to subtraction method. This was the case mainly for clusters in the nuclear region, Clump A, but also for a few clusters in Clumps $\mathrm{C}$ and $\mathrm{E}$. 


\subsection{Markarian 8}

For this galaxy we have F555W and F814W images. Cluster selection was done on the $\mathrm{F} 814 \mathrm{~W}$ image, but in all cases, the clusters are present in both images. Background subtraction was done using an annular region around each cluster.

\section{Conclusions}

We find that the starburst in NGC 7673 is very young, $<7 \mathrm{Myr}$, with some clusters possibly younger than $5 \mathrm{Myr}$. The starburst region is not concentrated, but is occuring in large, well-separated clumps. The starburst triggering mechanism for this galaxy must be able to explain widespread star formation. From the color-color diagrams it is apparent that reddening due to dust is only a significant factor in the nuclear region, Clump A.

An important question for NGC 7673 is how long the clumps will survive within the disk. It is unclear whether or not these large complexes of star formation will remain bound, which can be an important factor in galaxy evolution (see Noguchi 1999).

Our study of Markarian 8 is less conclusive, due to the fact that our filters do not cover a significant range in wavelength. However, it is apparent that the clusters in the clumps have a range of ages, and possibly masses. The region we call Disk 1 clearly shows the effect of reddening, the slope of which matches a Calzetti law for starburst galaxies rather than a standard CCM (Cardelli, Clayton, \& Mathis 1989) reddening law. Without an independent measure of cluster masses, additional broad band observations in the UV are needed to derive cluster ages. For both galaxies, study of the metallicity variations between the regions is also important for understanding broad band cluster analysis.

\section{References}

Ashman, K., \& Zepf, S. Globular Cluster Systems, Cambridge, UK: Cambridge University Press, 1998

Brodie, J., Schroder, L., Huchra, J., Phillips, A., Kissler-Patig, M., \& Forbes, D. 1998, AJ, 116, 691

Calzetti, D., Kinney, A., \& Storchi-Bergmann, T. 1996, ApJ, 458, 132

Cardelli, J., Clayton, G., \& Mathis, J. 1989, ApJ, 345, 245

Casini, C., \& Heidmann, J. 1976, A\&A, 47, 371

Dettmar, R., Heidmann, J., Klein, U., \& Wielebinski, R. 1984, A\&A, 130, 424

Ellis, R. 1997, ARA\&A, 35, 389

Elmegreen, B. 1997, ApJ, 486, 944

Guzman, R., Jangren, A., Koo, D., Bershady, M., \& Simard, L. 1998, ApJ, 495, L13

Holtzman J., et al. 1995, PASP, 107, 1065

Homeier, N. L., \& Gallagher, J. S. 1999, ApJ, 522, 199

Huchra, J. 1977, ApJS, 35, 171 
Hunter, D. A., O'Connell, R. W., Gallagher, J. S., Smecker-Hane, T. A. 2000, AJ, 120, 2383

Johnson, K. E., \& Conti, P. S. 2000, AJ, 120, 1273

Johnson, K. E., Leitherer, C., Vacca, W. D., Conti, P. S. 2000, AJ, 119, 2146

Leitherer, C. et al. 1999, ApJ, 123, 3

Noguchi, M. 1999, ApJ, 514, 77

Sargent, W. 1970, ApJ, 160, 405 